\title{
Caracterização e estudo da estabilidade física de suco misto adoçado de mangaba e cagaita
}

\author{
Herberth Diego Martins ${ }^{1}$, Danielle Godinho de Araújo Perfeito ${ }^{1}$, Anderson Rodrigo da Silva ${ }^{1}$, \\ Nei Peixoto ${ }^{2}$
}

${ }^{1}$ Instituto Federal Goiano, Campus Urutaí, Urutaí, Goiás, Brasil. E-mail: herberthdiego2703@ gmail.com, danielle.araujo@ifgoiano.edu.br, anderson.silva@ifgoiano.edu.br

${ }^{2}$ Universidade Estadual de Goiás, Unidade Universitária de Ipameri, Ipameri, Goiás, Brasil. E-mail: nei.peixoto48@gmail.com

Recebido: 06/03/2017; Aceito: 31/05/2017

\section{RESUMO}

O objetivo do trabalho consistiu em desenvolver e avaliar as características físico-químicas e sensoriais do suco tropical misto de mangaba e cagaita e verificar sua estabilidade física. Para isso, foram desenvolvidas três formulações padronizadas em $40 \%$ de polpa com as seguintes proporções: Formulação 1) $20 \%$ mangaba e $20 \%$ cagaita; Formulação 2) $10 \%$ mangaba e 30\% cagaita; e, Formulação 3) $30 \%$ mangaba e $10 \%$ cagaita. A caracterização físico-química dos sucos foi avaliada pelo teor de sólidos totais, acidez titulável, pH, sólidos solúveis e ratio. Para avaliação sensorial, cinquenta consumidores não treinados avaliaram aparência, aroma, sabor, textura e impressão global. Após a avaliação sensorial, a formulação de melhor aceitação foi analisada quanto à estabilidade física. Os resultados das análises físico-químicas das formulações foram satisfatórios, dentre os quais destaca-se o baixo $\mathrm{pH}$, valor desejável. As médias das notas atribuídas na análise sensorial classificaram todas as formulações como "gostei moderadamente". As três formulações foram aceitas pelos consumidores, sendo a formulação 1 escolhida para a realização do teste de estabilidade. $\mathrm{O}$ suco apresentou tendência à sedimentação, o aquecimento diminuiu ainda mais sua estabilidade. No tempo de 1 a 2,5 minutos de aquecimento a pectina impediu o efeito sob a desestabilização do suco.

Palavras-chave: fruto do cerrado, formação de sedimentos, viscosidade, hidrocoloide.

\section{Characterization and study of the physical stability of mangaba and cagaita's mixed juice}

\begin{abstract}
The objective was to develop and evaluate the physicochemical and sensorial characteristics of mangaba and cagaita mixed tropical juice and verify their physical stability. For this, three standard formulations were developed in $40 \%$ of pulp with the following proportions: Formulation 1) $20 \%$ mangaba and $20 \%$ cagaita; Formulation 2) 10\% mangaba and 30\% cagaita; and Formulation 3) 30\% mangaba and 10\% cagaita. The physicochemical characterization of the juice was evaluated by the total solids, titratable acidity, $\mathrm{pH}$, soluble solids and ratio. For sensory evaluation, fifty untrained consumers evaluated appearance, aroma, taste, texture and overall impression. After the sensory evaluation, the formulation of better acceptance was analyzed for physical stability. The results of the physicochemical analyzes of the formulations were satisfactory, among which the low $\mathrm{pH}$, desirable value, is outstanding. The averages of the scores attributed in the sensory analysis classified all formulations as "moderately liked". The three formulations were accepted by consumers, with formulation 1 being chosen for the stability test. The juice showed a tendency to sedimentation, warming decreased it is stability even more. In the time of 1 to 2.5 minutes of heating the pectin prevented the effect under destabilization of the juice.
\end{abstract}

Key words: Fruit of Cerrado, sediment formation, viscosity, hydrocolloid 


\section{Introdução}

O Bioma Cerrado apresenta aproximadamente $30 \%$ da biodiversidade de espécies vegetais brasileiras e 5\% da biodiversidade mundial. A sua flora é composta por mais de 11.627 espécies (MENDONÇA et al., 2008) e possui diversas plantas frutíferas com grande potencial econômico (SILVA et al., 2008). Além do aspecto nutritivo, as frutas do Cerrado são atrativas ao consumo pelas características sensoriais como variedade de sabor, textura e cor (SOUSA et al., 2010). Entre estas frutas destaca-se a mangaba (Hancoria speciosa Gomes) e a cagaita (Eugenia dysenterica DC.).

A mangabeira é uma fruteira pertencente à família Apocynaceae, nativa do Brasil, presente nas regiões Centro-Oeste, Sudeste, Norte e Nordeste nas áreas do cerrado e caatinga (VENTURINI FILHO, 2010). Os frutos são do tipo baga, de tamanho, forma e cores variados, geralmente elipsoidais ou arredondados, variando de 2,5 a $6,0 \mathrm{~cm}$ de diâmetro, exocarpo amarelado ou esverdeado com pigmentação vermelha ou sem pigmentação, polpa amarela, bastante suave, adocicada, carnoso-viscosa e ácida (GANGA et al., 2009; VENTURINI FILHO, 2010). O fruto apresenta boa digestibilidade e valor nutritivo, com teor de proteína superior à maioria das frutas comercializadas (VIEIRA NETO, 2001).

A cagaita pertence à família Myrtaceae (CARDOSO et al., 2011); o fruto da cagaiteira possui formato globoso, moderadamente achatado, casca frágil de coloração amarelo-claro e polpa com sabor agradável, discretamente ácida. A exploração tecnológica da cagaita mediante desenvolvimento de produtos alimentares pode representar alternativa de significância econômica, social e nutricional para as famílias do Cerrado brasileiro (BEDETTI et al., 2013). A cagaita pode ser utilizada na fabricação de diversos produtos. No entanto, devido às suas características químicas, como pH ácido, baixa acidez titulável e elevada umidade (CARDOSO et al., 2011), o fruto pode ser utilizado no desenvolvimento de bebidas, especialmente o suco (SILVA et al., 2008).

A junção de dois ou mais tipos de frutas resulta em produtos que se tornam mais atraentes pelo enriquecimento nas suas características nutritivas, principalmente em relação à diversidade e proporção de vitaminas e minerais. Além disso, colabora na melhoria das propriedades sensoriais. O objetivo desse estudo foi desenvolver e avaliar as características físico-químicas e sensoriais do suco misto de mangaba e cagaita, bem como verificar sua estabilidade física.

\section{Material e Métodos}

Elaboração do suco misto de mangaba e cagaita

Os frutos de mangaba e de cagaita correspondente à safra de 2015, foram adquiridos na Estação Experimental da Universidade Estadual de Goiás, Unidade Universitária de Ipameri. Os frutos foram transportados para a Unidade de Processamento de Frutas e Hortaliças do Instituto Federal Goiano - Campus Urutaí, onde foram selecionados, higienizados por imersão em solução de hipoclorito de sódio $\left(150 \mathrm{mg} \mathrm{L}^{-1}\right)$ por vinte minutos com posterior enxague e despolpados. As polpas obtidas foram embaladas à vácuo, identificadas e congeladas para posterior preparo do suco.

Para realização da pesquisa, foram desenvolvidas três formulações de suco misto com $40 \%$ de polpa. A formulação 1 caracterizou-se pela proporção $20 \%$ de polpa de mangaba e $20 \%$ de polpa de cagaita, sendo a formulação 2 composta por $10 \%$ de polpa de mangaba e $30 \%$ de polpa de cagaita e a formulação 3 composta por $30 \%$ de polpa de mangaba e $10 \%$ de polpa de cagaita.

O conteúdo de açúcar foi padronizado em $13 \%$ para todas as formulações. Os sucos foram preparados com a dissolução do açúcar em água sob agitação e posterior adição das polpas de mangaba e cagaita, previamente homogeneizadas. Os sucos foram aquecidos até atingirem a temperatura de $85{ }^{\circ} \mathrm{C}$ e imediatamente envasados a quente. As bebidas prontas foram acondicionadas em garrafas de vidro de $500 \mathrm{~mL}$, sanitizadas com solução de hipoclorito de sódio (50 $\mathrm{mg} \mathrm{L}^{-1}$ ), a quantidade suco vertida nas garrafas correspondeu $80 \%$ da capacidade da mesma. As garrafas foram invertidas, durante 5 minutos, para assegurar que o produto quente entrasse em contato com a parte superior da embalagem bem como esterilizar a tampa. As garrafas foram resfriadas, por imersão em água, imediatamente após o envase.

\section{Análises físico-químicas}

Para a caracterização físico-química e sensorial foi instalado o experimento em delineamento inteiramente casualizado, composto por três formulações, estabelecida como tratamentos. As análises físico-químicas foram realizadas no Laboratório de Análises Físico-químicas do Instituto Federal Goiano - Campus Urutaí, em triplicada, em que foram avaliados: teor de sólidos totais, determinado por secagem em estufa a $85{ }^{\circ} \mathrm{C}$, segundo o método $\mathrm{n}^{\circ} 920.151$ da AOAC (2010); acidez titulável, segundo o método $\mathrm{n}^{\circ} 942.15$ da AOAC (2010) e expressa em porcentagem de ácido cítrico; $\mathrm{pH}$, medido em potenciômetro calibrado com solução-tampão nos pHs 4 e 7 à $20{ }^{\circ} \mathrm{C}$, segundo o método $\mathrm{n}^{\circ} 981.12$ da AOAC (2010); sólidos solúveis ( $\left.{ }^{\circ} \mathrm{Brix}\right)$, baseado na leitura direta dos graus Brix da amostra a $20{ }^{\circ} \mathrm{C}$ em refratômetro digital; Ratio, quociente entre o teor de sólidos solúveis e acidez titulável.

\section{Análise sensorial}

A aparência, aroma, sabor, textura e impressão global das três formulações foram avaliadas por meio de análise sensorial. As análises foram realizadas no 
Laboratório de Análises Sensoriais do Instituto Federal Goiano - Campus Urutaí e consistiu na aplicação do teste de aceitação com escala hedônica estruturada de nove pontos, abrangendo de "desgostei muitíssimo" (1) a "gostei muitíssimo" (9). O teste foi realizado com 50 consumidores locados em cabines individuais, durante o período vespertino, em que degustaram de forma monádica e sequencial três amostras codificadas com números de três dígitos de forma balanceada e casualisada. Dessa forma, as três amostras apareceram o mesmo número de vezes em uma determinada posição, apresentadas sob luz artificial branca, em copos descartáveis brancos.

Os julgadores consumiram $30 \mathrm{~mL}$ de cada amostra a $7^{\circ} \mathrm{C}$, afim de não influenciar na emissão da opinião sobre o produto, além de permitir o consumo das demais amostras sem influenciar no julgamento. Os sucos foram mantidos em ambiente refrigerado durante a análise sensorial.

\section{Avaliação da estabilidade física}

Após a avaliação sensorial, a formulação de melhor aceitação foi analisada quanto à estabilidade física, por meio da visualização e medição da formação de sedimentos. O teste foi realizado com dois litros de sucos divididos em amostra $\mathrm{A}$, caraterizada pela não adição de estabilizante e a amostra $\mathrm{B}$, determinada pela adição de $0,01 \%$ de pectina, como hidrocoloide. As amostras, em duplicata, foram distribuídas, em tubos de ensaio devidamente identificados, até atingir a altura de líquido correspondente a $5 \mathrm{~cm}$. $\mathrm{O}$ ensaio foi submetido ao tratamento térmico em banho-maria a $85^{\circ} \mathrm{C}$, exceto o grupo controle ( $\mathrm{T} 0)$. Os demais permaneceram por 1 minuto ( $\mathrm{T}$ 1); 2,5 minutos ( $\mathrm{T}$ 2); 5 minutos ( $\mathrm{T}$ 3); 10 minutos (T 4); 25 minutos ( $\mathrm{T}$ 5) no banho-maria. Com o término de cada tratamento, os tubos foram acondicionados em estantes próprias, em repouso absoluto por período máximo de 48 horas.

A estabilidade física foi avaliada visualmente, medida pela quantidade de sedimento em cada tubo por meio de uma régua, logo após o tratamento térmico. $\mathrm{O}$ teste foi realizado imediatamente após o resfriamento da amostra e nos períodos 24 e 48 horas.

\section{Análises Estatísticas}

As variáveis acidez titulável, $\mathrm{pH}$, Ratio, sólidos solúveis, sólidos totais e os aspectos sensoriais de aparência, aroma, sabor, textura e impressão global foram submetidas a análise de variância seguida do Teste de Tukey.

Para os aspectos sensoriais, procedeu-se a análise de variáveis canônicas. Além disso, as notas de impressão global foram submetidas à análise de componentes principais, considerando os dados com variância unitária.
Quanto à avaliação da estabilidade física, foi instalado um experimento em delineamento inteiramente casualizado, em esquema fatorial $6 \times 3 \times 2$ (6 tempos de tratamento, 3 tempos de avaliação e 2 níveis de hidrocoloide), totalizando 36 tratamentos, com duas repetições. Cada unidade experimental foi constituída por um tubo de ensaio. As variáveis foram submetidas à análise de variância, ao teste de normalidade (Shapiro-Wilk) e posteriormente ao Teste de Scott-Knott. Foi adotado nível de 0,05 de significância em todos os testes, utilizando o software R (Core Development Core Team, 2016).

\section{Resultados e Discussão}

Análises físico-químicas

Constatou-se diferença estatística nos valores de acidez titulável, pH e sólidos solúveis (Tabela 1). Essas variações são justificadas pelas diferentes porcentagens de cada polpa (mangaba e cagaita) nas três formulações, visto que cada polpa tem suas características particulares.

Sobre a acidez titulável, a formulação 2, que possui maior proporção de cagaita, apresentou menor valor desse atributo. Isso pode ser explicado, pelo estudo desenvolvido por Perfeito et al. (2017) que mostra menor acidez titulável na polpa da cagaita quanto comparado à mangaba. Esses resultados são compatíveis com os obtidos por Cardoso et al. (2011)

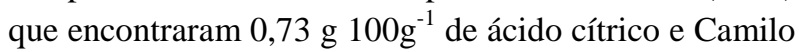

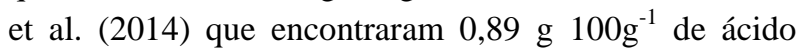
cítrico.

Em relação ao pH, valores abaixo de 4,5 limitam o crescimento de leveduras, fungos e bactérias sensíveis. Sob esse ponto de vista, as três formulações possuem essa característica positiva. Os sucos com maiores concentrações de mangaba, foram os que apresentam menores valores de $\mathrm{pH}$, que pode ser explicado pelas características físico-químicas intrínsecas do fruto.

O conteúdo de sólidos solúveis no fruto está relacionado ao teor de açúcares totais (CHITARRA; CHITARRA, 2005). Esse fato justifica os valores verificados no presente estudo, em que os sucos com maiores proporções de cagaita, possuem menor valores de sólidos solúveis. Aparentemente, por se tratar de plantas não domesticadas, variações nos parâmetros físico-químicos das polpas e consequentemente dos sucos estão previstas (NASCIMENTO et al., 2014).

\section{Análise sensorial}

Quanto à análise sensorial, observou-se que as três formulações não diferem numericamente ao teste de aceitação dos atributos aparência, aroma, sabor, textura e impressão global (Tabela 2). 
Tabela 1. Caracterização físico-química do suco misto de cagaita e mangaba

\begin{tabular}{llrr}
\hline \multicolumn{1}{c}{ Análise } & Formulação 1 & Formulação 2 & Formulação 3 \\
\hline Acidez titulável $\left(\mathrm{g} .100 \mathrm{~g}^{-1}\right)$ de ácido cítrico & $0,86^{\mathrm{a}} \pm 0,01$ & $0,80^{\mathrm{b}} \pm 0,02$ & $0,83^{\mathrm{ab}} \pm 0,02$ \\
$\mathrm{pH}$ & $3,50^{\mathrm{c}} \pm 0,02$ & $3,82^{\mathrm{a}} \pm 0,02$ & $3,64^{\mathrm{b}} \pm 0,02$ \\
Ratio & $15,05 \pm 0,20$ & $15,00 \pm 0,33$ & $15,66 \pm 0,38$ \\
Sólidos Solúveis ( ${ }^{\circ}$ Brix) & $13,00^{\mathrm{b}} \pm 0,00$ & $12,00^{\mathrm{a}} \pm 0,00$ & $13,00^{\mathrm{b}} \pm 0,00$ \\
Sólidos Totais $(\%)$ & $19,07 \pm 3,42$ & $17,06 \pm 1,51$ & $17,35 \pm 0,93$ \\
\hline
\end{tabular}

a, b, c iguais em uma mesma linha não diferem pelo teste de Tukey ( $>0,05)$.

Tabela 2.Valores referentes a análise sensorial do suco misto de mangaba e cagaita

\begin{tabular}{llll}
\hline Atributo & Formulação 1 & Formulação 2 & Formulação 3 \\
\hline Aparência & $7,36 \pm 1,41$ & $7,28 \pm 1,30$ & $7,22 \pm 1,42$ \\
Aroma & $6,80 \pm 1,87$ & $7,04 \pm 1,55$ & $7,06 \pm 1,62$ \\
Sabor & $7,10 \pm 1,71$ & $6,80 \pm 1,98$ & $7,16 \pm 1,69$ \\
Textura & $7,42 \pm 1,43$ & $7,12 \pm 1,81$ & $7,30 \pm 1,27$ \\
Impressão Global & $7,14 \pm 1,73$ & $7,20 \pm 1,59$ & $7,22 \pm 1,42$ \\
\hline
\end{tabular}

A maioria das médias dos atributos estão próximos à nota 7 que corresponde ao termo "gostei moderadamente" na escala hedônica estruturada, indicando que a associação dos dois frutos foi bem aceita. Apesar de serem frutos típicos do Cerrado, ainda é pequeno o consumo de mangaba e cagaita pela população em geral; o desconhecimento dos aspectos organolépticos dos frutos pode ter sido um fator que gerou a resposta de aceitação sensorial dos sucos. A média aceitável dos atributos torna-se importante para uma futura inserção do produto no mercado. As médias de aceitação para impressão global foram próximas aos encontrados por Assumpção et al. (2013), que obtiveram média para esse atributo em néctar misto de mangaba e cagaita de 7,96.

A representação gráfica da Análise de componentes principais está apresentada na Figura 1. A diferença entre as respostas dos consumidores pode ser observada pela localização de cada uma no gráfico. Verificou-se que $58,6 \%$ da variação obtida entre as respostas dos consumidores foi explicada pelo eixo Componente Principal 1 e 25,2\% pelo eixo Componente Principal 2. Os Componentes Principais 1 e 2 somados justificaram $83,8 \%$ da variação entre as notas dadas pelos julgadores.

A figura 1 mostra os vetores ilustrados das três formulações. As notas dadas pelos consumidores estão mais agrupadas próximas aos vetores das formulações 1,2 e 3 . Por meio da difusão das respostas nos eixos representativos dos dois componentes principais considerados, é possível avaliar a similaridade ou dissimilaridade das formulações dos sucos.

A formulação 2 associou-se negativamente aos dois componentes principais. As formulações 1 e 3, por sua vez, relacionaram-se positivamente com o componente principal 2, porém, o comprimento do vetor da formulação 3 foi menor do que o produzido pela formulação 1, indicando que as notas da formulação 1 tiveram maior variabilidade. Apesar de estarem próximos, o que indica certa similaridade, o maior comprimento do vetor da formulação 1, sugere tendência de aceitação da maioria dos consumidores, o que leva a concluir que a formulação 1 foi a mais bem aceita.

Na Figura 2, é possível comparar as formulações pela proximidade das elipses apresentadas. Dessa forma, observa-se que não houve sobreposição entre as áreas abrangidas pelas elipses de cada formulação. Isso significa que, houve atributos sensoriais diferentes que melhor caracterizaram cada uma das formulações.

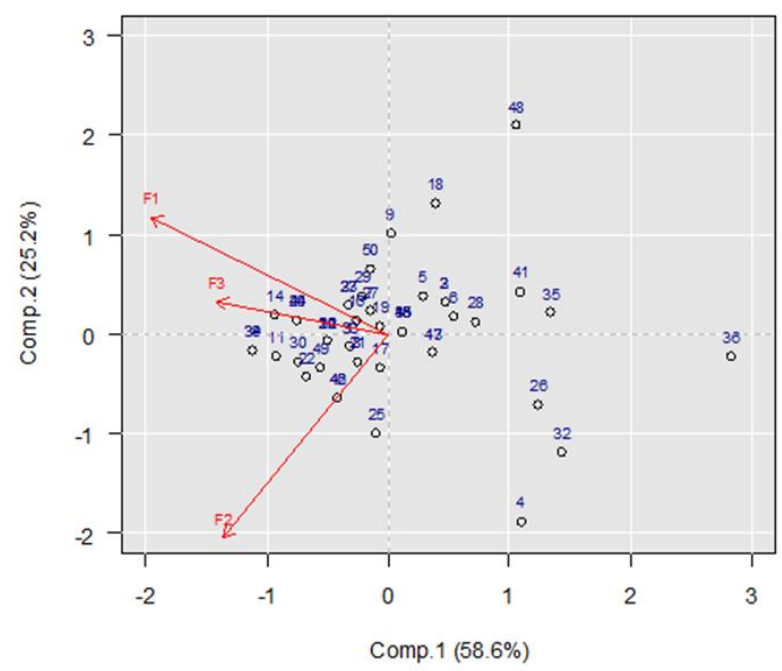

Figura 1. Biplot contendo escores dos dois primeiros componentes principais e cargas das formulações a partir de notas de impressão global do suco misto de mangaba e cagaita. 
Quanto mais longo o vetor e mais próximos ao centro do intervalo de confiança, maior é a importância do atributo na diferenciação das formulações. A formulação 1 caracterizou-se melhor pela textura, enquanto que a formulação 2 foi marcada pelo aroma. A formulação 3 manteve-se mediana. Notou-se que pelo menor comprimento do vetor, a impressão global foi pouco relevante para diferenciação das formulações.

\section{Estabilidade física}

Dada a melhor aceitação da formulação 1, foi realizado o teste de estabilidade física no suco composto por $20 \%$ de polpa de mangaba e $20 \%$ de polpa de cagaita. Os resultados da ANOVA para estabilidade estão apresentados na Tabela 3.

Os resultados apontam para uma interação significativa entre o tempo de tratamento térmico e a presença do hidrocoloide, além das diferenças obtidas individualmente em relação às duas fontes de variação.

$\mathrm{O}$ intervalo entre o resfriamento do suco e a avaliação da estabilidade não alterou os resultados, independentemente da presença ou não do hidrocoloide ou o tempo de tratamento térmico. Sob o ponto de vista industrial, os resultados sugerem que o teste de estabilidade para a formulação 1 independe do tempo de armazenamento do suco. A Tabela 4 mostra a comparação entre as médias das combinações entre o uso do hidrocoloide e o tempo de tratamento térmico.

O teste de estabilidade física está relacionado, dentre outros fatores, ao grau de viscosidade. Segundo BEZERRA et al. (2009) alguns fatores podem influenciar a viscosidade de derivados de frutas como o tipo de fruta e o teor de sólidos como açúcares, pectina e fibras. De acordo com SILVA et al. (2005) a concentração da polpa ou suco de fruta também pode ter efeito sobre a viscosidade. Sendo assim, esperava-se a formação de sedimento, mesmo sem a adoção de medidas que alterassem a estabilidade do suco.

As médias de sedimentação obtidas com ou sem a presença do hidrocoloide, sem o tratamento térmico, foram semelhantes. Isso indica que a pectina foi ineficaz em impedir a sedimentação. Entretanto, ela se mostrou bastante eficiente, quando aplicado o tratamento térmico por um período entre um minuto e 2,5 minutos. Após esse período de aquecimento, a pectina não foi capaz de impedir a formação de sedimento.

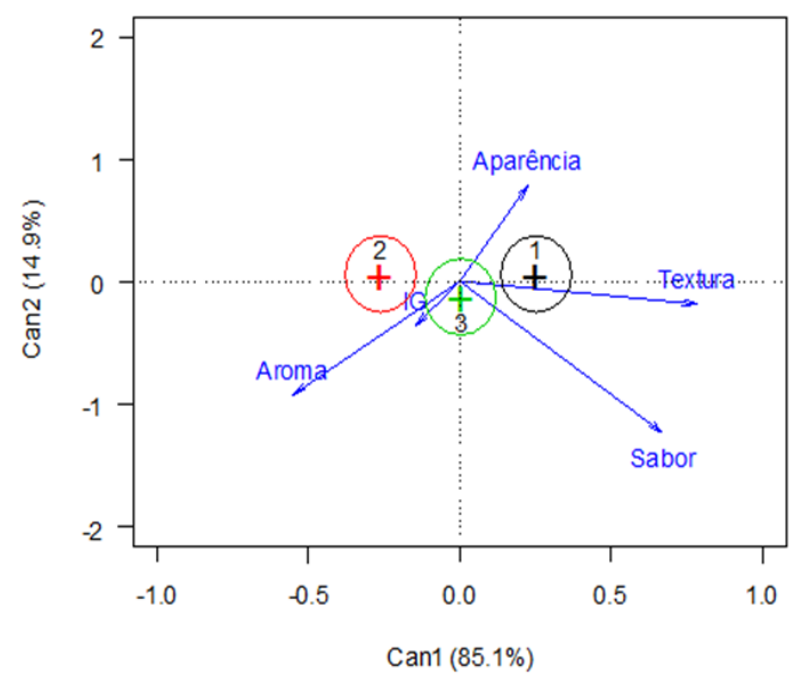

Figura 2. Biplot contendo elipses de $95 \%$ de confiança dos escores médios das duas variáveis canônicas a partir de atributos sensoriais de três formulações.

Tabela 3. Análise de variância da formação de sedimentos no suco misto de mangaba e cagaita

\begin{tabular}{|c|c|c|c|c|c|}
\hline Fonte de variação & $\begin{array}{c}\text { Grau de } \\
\text { Liberdade }\end{array}$ & $\begin{array}{l}\text { Soma do } \\
\text { Quadrado }\end{array}$ & Quadrado Médio & Teste F & $\mathrm{p}$ valor \\
\hline Tempo de Tratamento & 5 & 17646 & 3529,1 & 24,3526 & $<0,001 * * *$ \\
\hline Tempo de Avaliação & 2 & 71,6 & 35,8 & 0,247 & 0,782 \\
\hline Hidrocoloide & 1 & 734,7 & 734,7 & 5,07 & $0,03 *$ \\
\hline $\begin{array}{l}\text { Tempo de Tratamento x Tempo de } \\
\text { Avaliação }\end{array}$ & 10 & 593,4 & 59,3 & 0,4095 & 0,933 \\
\hline $\begin{array}{l}\text { Tempo de Tratamento } \mathrm{x} \\
\text { Hidrocoloide }\end{array}$ & 5 & 3641 & 728,3 & 5,0256 & $0,001 * *$ \\
\hline $\begin{array}{l}\text { Tempo de Avaliação x } \\
\text { Hidrocoloide }\end{array}$ & 2 & 352,2 & 176,1 & 1,2152 & 0,308 \\
\hline $\begin{array}{l}\text { Tempo de Tratamento x Tempo de } \\
\text { Avaliação x Hidrocoloide }\end{array}$ & 10 & 472,1 & 47,2 & 0,3258 & 0,968 \\
\hline Resíduo & 36 & 5271 & 144,9 & & \\
\hline $\begin{array}{l}\text { Média (Rank) } \\
\text { CV\% }\end{array}$ & & & $\begin{array}{c}0,261(36,5) \\
32,97\end{array}$ & & \\
\hline
\end{tabular}

A análise foi realizada com os Ranks dos dados originais, devido à falta de normalidade. 
Tabela 4. Comparação de médias de tratamentos: combinações de tempo de tratamento, tempo de avaliação e hidrocoloide relacionado à formação de sedimentos

\begin{tabular}{ccc}
\hline $\begin{array}{c}\text { Tempo de } \\
\text { Tratamento }\end{array}$ & Hidrocoloide & $\begin{array}{c}\text { Rank da formação de } \\
\text { Sedimentos } \\
\text { (valores médios reais) }\end{array}$ \\
\hline 0 & SEM & $14,5(0,00) \mathrm{c}$ \\
0 & COM & $14,5(0,00) \mathrm{c}$ \\
1 & SEM & $35,83(0,27) \mathrm{b}$ \\
1 & COM & $14,5(0,00) \mathrm{c}$ \\
2 & SEM & $29,83(0,22) \mathrm{c}$ \\
2 & COM & $19,5(0,07) \mathrm{c}$ \\
3 & SEM & $49,16(0,42) \mathrm{b}$ \\
3 & COM & $46,83(0,40) \mathrm{b}$ \\
4 & SEM & $68(0,58) \mathrm{a}$ \\
4 & COM & $44,83(0,98) \mathrm{b}$ \\
5 & SEM & $40,83(0,30) \mathrm{b}$ \\
5 & COM & $59,66(0,50) \mathrm{a}$ \\
\hline
\end{tabular}

Médias seguidas de mesma letra na coluna não diferem pelo teste Scott-Knott $(p>0,05)$.

O tratamento térmico foi capaz de alterar a sedimentação do suco sem o hidrocoloide desde o menor período de aquecimento. Isso pôde ser comprovado também pela grande significância $(\mathrm{p}<0,001)$ verificada na Tabela 3. No caso de sucos de frutas ou polpas, a viscosidade aparente diminui à medida que se eleva a temperatura, já que, a maioria destes produtos apresenta-se na forma de sólidos dispersos em meio líquido e um aumento da temperatura ocasiona diminuição da viscosidade da fase fluida aumentado a mobilidade das partículas em suspensão (FERREIRA, 2008).

\section{Conclusões}

O suco misto de mangaba e cagaita apresentou boa aceitação sensorial, principalmente na formulação com $20 \%$ de polpa de mangaba e $20 \%$ de polpa de cagaita. Sob essa formulação, o suco apresenta tendência à sedimentação mesmo sem tratamento térmico. Com emprego do tratamento térmico a sedimentação aumenta, porém, nos tempos de 1 e 2,5 minutos de aquecimento a pectina conseguiu diminuir o efeito do tempo de aquecimento na desestabilização do suco. Sugere-se então para este tipo de suco o método de pasteurização rápida.

\section{Referências Bibliográficas}

AOAC. ASSOCIATION OF OFFICIAL ANALYTICAL CHEMISTS. Official methods of analysis. Gaithersburg (Md.), revision 3, 2010. C.; MORZELLE, M. C.; VILAS BOAS, B. M., DE SOUZA,
E. C. Néctar misto de mangaba (Hancoria speciosa Gomes) e cagaita (Eugenia dysenterica): perfil sensorial e características físico-químicas. Revista Brasileira de Produtos Agroindustriais, Campina Grande - PB, v. 15, n. 3, p. 219 224, 2013

BEDETTI, S. F.; CARDOSO, L. M.; SANTOS, P. R. G.; DANTAS, M. I. S.; PINHEIRO-SANT'ANA, H. M. Néctar de cagaita (Eugenia dysenterica DC.): desenvolvimento, caracterização microbiológica, sensorial, química e estudo da estabilidade. Boletim CEPPA, Curitiba-PR, v. 31, n. 1, p. 125-138, 2013.

BEZERRA, J. R. M. V.; RIGO, M.; DEMCZUK J. B.; CÓRDOVA, K.R.V. Estudo do Efeito da Temperatura nas Propriedades Reológicas da Polpa de Morango (Fragaria ananassa). Ambiência - Revista do Setor de Ciências Agrárias e Ambientais, Guarapuava-PR, v. 5 n. 1 p. 37- 47, 2009.

CAMILO, Y. M. V.; SOUZA, E. R. B.; VERA, R.; NAVES, R. V. Caracterização de frutos e seleção de progênies de cagaiteiras (Eugenia dysenterica DC.). Científica, Jaboticabal-SP, v. 42, n. 1, p. 1-10, 2014.

CARDOSO, L. M.; MARTINO, H. S. D.; MOREIRA, A. V. B.; RIBEIRO, S. M. R.; PINHEIRO-SANT'ANA, H. M. Cagaita (Eugenia dysenterica DC.) of the Cerrado of Minas Gerais, Brazil: Physical and chemica characterization, carotenoids and vitamins. Food Research International. Viçosa-MG, v. 44, n 7, p. 2151-2154, 2011.

ChITARrA, M. I. F.; CHITARRA, A. B. Pós-colheita de frutos e hortaliças: fisiologia e manuseio. 2 ed., Lavras-MG: UFLA, 2005, $783 \mathrm{p}$.

FERREIRA, G. M.; GUIMARÃES, M. J. O.; MAIA, M. C. Efeito da Temperatura e taxa de cisalhamento nas propriedades de escoamento da polpa de capuaçu (T. grandiflorum Schum) integral. Revista Brasileira de Fruticultura, Jaboticabal-SP, v. 30, n. 2, p. 385-389, 2008.

GANGA, R. M. D.; CHAVES, L. J.; NAVES, R. V. Parâmetros genéticos em progênies de Hancornia speciosa Gomes do Cerrado. Scientia Forestalis, Piracicaba-SP, v. 37, n. 84 , p. $395-404,2009$.

MENDONÇA, R. C.; FELFILI, J. M.; WALTER, B. M. T.; SILVA JÚNIOR, M. C.; REZENDE, A. V.; FILGUEIRAS, T. S.; NOGUEIRA, P. E. E FAGG, C.W. Flora Vascular do Bioma Cerrado - Checklist com 12.356 espécies. In: SANO, S. M.; ALMEIDA, S. P.; RIBEIRO, J. F. (Eds.). Cerrado: ambiente e flora. 2. ed. Planaltina-SP: Embrapa Cerrados, 2008, p. 421-443.

NASCIMENTO, R. S. M.; CARDOSO, J. A.; COCOZZA, F. D. M. Caracterização física e físico-química de frutos de mangabeira (Hancornia speciosa Gomes) no oeste da Bahia. Revista Brasileira de Engenharia Agrícola Ambiental, Campina Grande-PB, v. 18 n. 8, p. 856-860, 2014.

PERFEITO, D. G. A.; CORRÊA, I. M.; PEIXOTO, N. Elaboração de bebida com extrato hidrossolúvel de soja saborizada com frutos do cerrado. Revista de Agricultura Neotropical, Cassilândia-MS, v. 4, n. 1, p. 22-28, 2017.

SILVA, F. C.; GUIMARÃES, D. H. P.; GASPARETTO, C. A. Reologia do Suco de Acerola: Efeitos da Concentração e 
Temperatura. Ciência de Tecnologia de Alimentos, Campinas-SP, v. 25, n. 1, p. 121-126, 2005.

SILVA, M. R.; LACERDA, D. B. C. L.; SANTOS, G. G. E MARTINS, D. M. O. Caracterização química de frutos nativos do Cerrado. Ciência Rural, Santa Maria-RS, v. 38, n. 6, p. 1790-1793, 2008.

SOUSA, P. H. M.; MAIA, G. A.; AZEREDO, H. M. C.; RAMOS, A. M.; FIGUEIREDO, R. W. Storage stability of a tropical fruit (cashew apple, acerola, papaya, guava and passion fruit) mixed néctar added caffeine. International Journal of Food Science and Technology, Londres, v. 45, n. 10, p. 2162-2166, 2010.
SOUZA FILHO, M. S. M. S.; LIMA, J. R.; NASSU, R. T.; MOURA, C. F. H.; BORGES, M. D. F. Formulações de néctares de frutas nativas das regiões norte e nordeste do Brasil; Boletim CEPPA, Curitiba- PR, v. 18, n. 2, p. 275-283, 2000.

VENTURINI FILHO, W. G. Bebidas alcoólicas - Ciência e tecnologia. São Paulo-SP: Blucher. 2010. 461p.

VIEIRA NETO, R. D. Recomendações técnicas para o cultivo da mangabeira, Aracaju-SE: Embrapa Tabuleiros Costeiros, 2001. 26 p. (Circular Técnica, 20). 